\title{
THE INDEPENDENCE OF GAME THEORY \\ OF UTILITY THEORY
}

\author{
BY BEZALEL PELEG
}

\section{Communicated by J. Milnor, April 25, 1966}

A theory of noncooperative and cooperative games, that parallels the classical theory [1], [2] but makes no use of utility theory, is outlined in this note.

1. Games in normal form. The following definition seems suitable for our purpose (see, e.g., $[3, \S 5]$ ).

Definition 1.1. An n-person game (in normal form) is a system $G=\left\{N ; S^{1}, \cdots, S^{n} ; X^{1}, \cdots, X^{n} ; R^{1}, \cdots, R^{n} ; H^{1}, \cdots, H^{n}\right\}$, where:

(1.1) $N$ is a set of $n$ members (the players of $G$ ), and for each $i \in N$ :

(1.2) $S^{i}$ is a nonempty set (the set of strategies of player $i$ ).

(1.3) $X^{i}$ is a nonempty set (the set of outcomes for player $i$ ).

(1.4) $\quad R^{i} \subset X^{i} \times X^{i}$ (the preference relation of player $i$ ).

(1.5) $H^{i}$ is a function whose domain is the set $S=S^{1} \times \cdots \times S^{n}$ and whose range is $X^{i}$ (the payoff function of player $i$ ).

If $\bar{s} \in S, \bar{s}=\left(\bar{s}^{1}, \cdots, \bar{s}^{n}\right)$, and $s^{i} \in S^{i}$, we denote:

$$
\bar{s} \mid s^{i}=\left(\bar{s}^{1}, \cdots, \bar{s}^{i-1}, s^{i}, \bar{s}^{i+1}, \cdots, \bar{s}^{n}\right)
$$

Definition 1.2. Let $G=\left\{N ; S^{1}, \cdots, S^{n} ; X^{1}, \cdots, X^{n} ; R^{1}, \cdots\right.$, $\left.R^{n} ; H^{1}, \cdots, H^{n}\right\}$ be an $n$-person game. $\bar{s} \in S$ is an equilibrium point for $G$ if for each $i \in N$ :

$$
\left(H^{i}\left(\bar{s} \mid s^{i}\right), H^{i}(\bar{s})\right) \notin R^{i}, \quad \text { for all } s^{i} \in S^{i} .
$$

This is Nash's definition [2] adjusted to our case.

2. Finite, noncooperative games. Let

$$
G=\left\{N ; S^{1}, \cdots, S^{n} ; X^{1}, \cdots, X^{n} ; R^{1}, \cdots, R^{n} ; H^{1}, \cdots, H^{n}\right\}
$$

be an $n$-person game. $G$ is finite if $S^{i}$ is finite for all $i \in N$. The mixed extension of ${ }^{1} G$ is the $n$-person game

$$
\hat{G}=\left\{\hat{N} ; \hat{S}^{1}, \cdots, \hat{S}^{n} ; \hat{X}^{1}, \cdots, \hat{X}^{n} ; \hat{R}^{1}, \cdots, \hat{R}^{n} ; \hat{H}^{1}, \cdots, \hat{H}^{n}\right\},
$$

where:

$$
\hat{N}=N,
$$

\footnotetext{
${ }^{1}$ In what follows we assume that $G$ is finite.
} 
and for each $i \in N$ :

(2.2) $\hat{S}^{i}$ is the set of all probability distributions on $S^{i}$ (the mixed strategies of player $i$ ).

(2.3) $\hat{X}^{i}$ is a set of probability distributions on $X^{i}$, specifically the range of $\hat{H}^{i}$ (see (2.5)).

(2.4) $\hat{R}^{i} \subset \hat{X}^{i} \times \hat{X}^{i} ;$ and

(2.5) $\hat{H}^{i}$ is a function which assigns to each $\hat{s} \in \hat{S}=\hat{S}^{1} \times \cdots \times \hat{S}^{n}$ a probability distribution on $X^{i}$, where the probability of $H^{i}\left(s^{1}, \cdots, s^{n}\right)$ according to $\hat{H}^{i}(\hat{s})$ is

$$
\sum_{\left\{s_{1}: H^{i}\left(s_{1}\right)=H^{i}(s)\right\}} \hat{s}^{1}\left(s_{1}^{1}\right) \cdots \hat{s}^{n}\left(s_{1}^{n}\right)
$$

for all $s=\left(s^{1}, \cdots, s^{n}\right) \in S$.

It is clear that each of the sets $\hat{S}^{1}, \cdots, \hat{S}^{n}$, or $\hat{X}^{1}, \cdots, \hat{X}^{n}$ can be considered as a subset of a suitable euclidean space; this assumption will be made from now on.

THEOREM 2.1. If for each $i \in N$ :

(2.6) The relation $\hat{R}^{i}$ is acyclic (see $[1$, p. 591]);

(2.7) $\hat{R}^{i}$ is open (in the relative topology of $\hat{X}^{i} \times \hat{X}^{i}$ );

(2.8) if $x, y, z \in \hat{X}^{i}, 0 \leqq \alpha \leqq 1$, and $\alpha y+(1-\alpha) z \in \hat{X}^{i}$ then:

(2.8.1) if $(x, y) \notin \hat{R}^{i}$ and $(x, z) \notin \hat{R}^{i}$ then $(x, \alpha y+(1-\alpha) z) \notin \hat{R}^{i}$; and

(2.8.2) if $(y, x) \notin \hat{R}^{i}$ and $(z, x) \notin \hat{R}^{i}$ then $(\alpha y+(1-\alpha) z, x) \notin \hat{R}^{i}$, then $\hat{G}$ has an equilibrium point.

We shall need the following result [4] in the proof of Theorem 2.1.

THEOREM 2.2. If for each $\hat{s} \in \hat{S}$ and for each $i \in N$ a binary relation $Z^{i}(\hat{s})$ is defined on $S^{i}$ such that:

(2.9) $Z^{i}(\hat{s})$ is acyclic.

(2.10) if $s_{h}^{i}, s_{k}^{i} \in S^{i}$ then $\left\{\hat{s}:\left(s_{h}^{i}, s_{k}^{i}\right) \in Z^{i}(\hat{s})\right\}$ is open (in the relative topology of $\hat{S})$; and

(2.11) if $s_{k}^{i} \in S^{i}$ and $\hat{s}^{i}\left(s_{k}^{i}\right)=0$ then there exists no $s_{h}^{i} \in S^{i}$ such that $\left(s_{h}^{i}, s_{k}^{i}\right) \in Z^{i}(\hat{s})$,

then there exists $\hat{s}_{0} \in \hat{S}$ such that $Z^{i}\left(\hat{s}_{0}\right)=\varnothing$ for all $i \in N$.

Proof of Theorem 2.1. For each $\hat{s} \in \hat{S}$ and for each $i \in N$ we define a binary relation $Z^{i}(\hat{s})$ on $S^{i}$ by:

$$
\left(s_{h}^{i}, s_{k}^{i}\right) \in Z^{i}(\hat{s}) \Leftrightarrow\left(\hat{H}^{i}\left(\hat{s} \mid s_{h}^{i}\right), \hat{H}^{i}\left(\hat{s} \mid s_{k}^{i}\right)\right) \in \hat{R}^{i} \quad \text { and } \quad \hat{s}^{i}\left(s_{k}^{i}\right)>0 .
$$

The validity of (2.9), (2.10) and (2.11) for $Z^{i}(\hat{s})$ follows from (2.6), (2.7) and (2.12). By Theorem 2.2 there exists $\hat{s}_{0} \in \hat{S}$ such that $Z^{i}\left(\hat{s}_{0}\right)=\varnothing$ for all $i \in N$. We claim that $\hat{s}_{0}$ is an equilibrium point for $\hat{G}$. Let $i \in N$. If $\hat{s}_{0}^{i}\left(s_{k}^{i}\right)>0$ then 


$$
\left(\hat{H}^{i}\left(\hat{s}_{0} \mid s_{h}^{i}\right), \hat{H}^{i}\left(\hat{s}_{0} \mid s_{k}^{i}\right)\right) \notin \hat{R}^{i} \text { for all } s_{h}^{i} \in S^{i} .
$$

Hence, by (2.8.2), $\left(\hat{H}^{i}\left(\hat{s}_{0} \mid \hat{s}^{i}\right), \hat{H}^{i}\left(\hat{s}_{0} \mid s_{k}\right)\right) \notin \hat{R}^{i}$ for all $\hat{s}^{i} \in \hat{S}^{i}$. Since

$$
\hat{H}^{i}\left(\hat{s}_{0}\right)=\sum_{\substack{\left.i \\ l_{s_{k}}^{i}: \hat{s}_{0}^{i}\left(s_{k}^{i}\right)>0\right\}}} \hat{s}_{0}^{i}\left(s_{k}^{i}\right) \hat{H}^{i}\left(\hat{s}_{0} \mid s_{k}^{i}\right)
$$

it follows from (2.8.1) that $\left(\hat{H}^{i}\left(\hat{s}_{0} \mid \hat{s}^{i}\right), \hat{H}^{i}\left(\hat{s}_{0}\right)\right) \notin \hat{R}^{i}$ for all $\hat{s}^{i} \in \hat{S}^{i}$. This completes the proof.

We remark that the classical case, where:

(2.13) each of the relations $\hat{R}^{i}$ is derived from a complete transitive relation $\bar{R}^{i} \subset \hat{X}^{i} \times \hat{X}^{i}$ by:

(2.13.1) $\quad \hat{R}^{i}=\left\{(x, y):(x, y) \in \bar{R}^{i} \quad\right.$ and $\left.\quad(y, x) \notin \bar{R}^{i}\right\} ;$ and

(2.14) there exists a real function (a utility) $u^{i}$ defined on $\hat{X}^{i}$ which satisfies:

(2.14.1) $\quad u^{i}(x) \geqq u^{i}(y) \Leftrightarrow(x, y) \in \bar{R}^{i}$; and

(2.14.2) $\quad u^{i}(\alpha x+(1-\alpha) y)=\alpha u^{i}(x)+l(1-\alpha) u^{i}(y)$, for all $x, y \in \hat{X}^{i}$ and for all $0 \leqq \alpha \leqq 1$ such that $\alpha x+(1-\alpha) y \in \hat{X}^{i}$,

is contained in our result. To see that the inclusion is strict, consider the following example:

EXAMPLE 2.3. Let $\hat{X}$ be the simplex

$$
\left\{a: a=\left(a_{1}, a_{2}, a^{8}\right), a_{1} \geqq 0, a_{2} \geqq 0, a_{3} \geqq 0 \text { and } a_{1}+a_{2}+a_{3}=1\right\} \text {. }
$$

Define a relation $\bar{R}$ by:

$$
\left(a, a^{\prime}\right) \in \bar{R} \Leftrightarrow \frac{a_{3}}{a_{1}+2 a_{2}+a_{3}} \geqq \frac{a_{3}^{\prime}}{a_{1}^{\prime}+2 a_{2}^{\prime}+a_{3}^{\prime}} .
$$

There exists no utility function on $\hat{X}$ which represents $\bar{R}$ in the sense of (2.14), while the relation $\hat{R}$, derived from $\bar{R}$ by (2.13.1) satisfies (2.6), (2.7) and (2.8). (This example is a modification of an example of R. J. Aumann.)

We plan also to discuss the nonclassical approaches to utility theory [5], [3].

3. Two-person zero-sum games. In this section we use our notation to formulate some well-known properties of two-person zerosum games (see, e.g., [6, Chapter 4]). Let $G=\left\{\{1,2\} ; S^{1}, S^{2} ; X^{1}, X^{2}\right.$; $\left.R^{1}, R^{2} ; H^{1}, H^{2}\right\}$ be a two-person game.

DEFINITION 3.1. $G$ is zero-sum if:

$$
\left(H^{1}\left(s_{1}\right), H^{1}\left(s_{2}\right)\right) \in R^{1} \Leftrightarrow\left(H^{2}\left(s_{2}\right), H^{2}\left(s_{1}\right)\right) \in R^{2}, \quad \text { for all } s_{1}, s_{2} \in S .
$$


We remark that if both ${ }^{2} R^{1}$ and $R^{2}$ are complete orders then every pair of equilibrium points $s_{1}$ and $s_{2}$ for $G$ are equivalent, i.e., $\left(H^{1}\left(s_{1}\right)\right.$, $\left.H^{2}\left(s_{1}\right)\right)=\left(H^{1}\left(s_{2}\right), H^{2}\left(s_{2}\right)\right)$, and interchangeable, i.e., $\left(s_{1}^{1}, s_{2}^{2}\right)$ and $\left(s_{2}^{1}, s_{1}^{2}\right)$ are also equilibrium points $[6$, p. 106].

4. Cooperative games. An important step towards the liberation of the theory of cooperative games from utility theory was made in [7]. Pushing it further we define:

DEFINITION 4.1. An $n$-person game in characteristic function form is a system $G=\left\{N ; X^{1}, \cdots, X^{n} ; R^{1}, \cdots, R^{n} ; v\right\}$ where:

(4.1) $N$ is a set with $n$ members (the players of $G$ ).

(4.2) for each $i \in N, X^{i}$ is a nonempty set (the set of payoffs for player $i)$.

(4.3) for each $i \in N, R^{i} \subset X^{i} \times X^{i}$ (the preference relation of player $i$ ).

(4.4) $v$ is a function which assigns to each subset $S \subseteq N$ a set $v(S) \subset X$ $=X^{1} \times \cdots \times X^{n}$, (the characteristic function).

A "dominance relation" can now be defined on $X$ :

Definition 4.2 . Let $x=\left(x^{1}, \cdots, x^{n}\right)$ and $y=\left(y^{1}, \cdots, y^{n}\right)$ belong to $X . x$ dominates $y$ via a subset $S \subseteq N$ if :

$$
\begin{gathered}
x \in \vartheta(S) ; \text { and } \\
\left(x^{i}, y^{i}\right) \in R^{i} \text { for all } i \in S .
\end{gathered}
$$

It is now possible to define solutions [1], the core $[8, \mathrm{p} .1], \psi$ stable pairs [6, Chapter 10] and bargaining sets [9] of a game in a characteristic function form. Some other notions, such as value [10] and kernel [11], might be more difficult to generalize, but still can be defined for restricted classes of cooperative games.

\section{Remarks.}

REMARK 5.1. It is possible to define games in extensive form within the framework of our suggested approach (see, e.g., [6, p. 44]). The normal form can be obtained from the extensive form, and the characteristic function form from the normal form, as in the classical theory.

REMARK 5.2. The generalization of our definitions to games with infinite number of players [12], [13] is straightforward. Actually, our definitions 4.1 and 4.2 are contained, at least implicitly, in the above references.

${ }^{2} \mathrm{By}(3.1)$ it is sufficient to assume that $R^{1}$ (or $R^{2}$ ) is a complete order. 


\section{REFERENCES}

1. J. von Neumann and O. Morgenstern, Theory of games and economic behavior, Princeton Univ. Press, Princeton, N. J., 1943, 3rd ed., 1953.

2. J. F. Nash, Non-cooperative games, Ann. of Math. 54 (1951), 286-295.

3. R. J. Aumann, Utility theory without the completeness axiom, Econometrica 30 (1962), 445-462.

4. B. Peleg, Equilibrium points for open acyclic relations, Canad. J. Math. (to appear).

5. M. Hausner, Multidimensional utilities, Decision processes, Wiley, New York, 1954, pp. $167-180$.

6. R. D. Luce and H. Raiffa, Games and decisions, New York, Wiley, 1957.

7. R. J. Aumann and B. Peleg, von Neumann-Morgenstern solutions to co-operative games without side payments, Bull. Amer. Math. Soc. 66 (1960), 173-179.

8. L. S. Shapley and M. Shubik, The core of an economy with nonconvex preferences, The Rand Corporation, Santa Monica, California, R-M=3518-PR, 1963.

9. R. J. Aumann and M. Maschler, The bargaining set for cooperative games, Advances in Game Theory, Princeton Univ. Press, Princeton, N. J., 1964, pp. 443-476.

10. L. S. Shapley, $A$ value for n-person games, Contributions to the Theory of Games II, Princeton Univ. Press, Princeton, N. J., 1953, pp. 307-318.

11. M. Davis and M. Maschler, The kernel of a cooperative game, Econometric Research Program R-M 58, Princeton University, Princeton, N. J., 1963.

12. G. Debreu and H. Scarf, $A$ limit theorem on the core of an economy, International Economic Review, 4 (1963), 235-246. 39-50.

13. R. J. Aumann, Markets with a continuum of traders, Econometrica 32 (1964),

HEBREW UNIVERSITY

JERUSALEM, ISRAEL 